Edygarova feststellt, ist die Standardsprache für viele eine fremde Sprachform, denn sie wird selten verwendet, in der Schule unzureichend gelehrt, und die Sprecher haben keine emotionale Bindung an sie. Aus demselben Grund werden auch viele in den letzten Jahrzehnten geschaffene Neologismen abgelehnt. Im Hinblick auf die Erhaltung der Sprache vertritt diese Einstellung eine falsche Strategie: Wenn man den Sprechern eine fehlerhafte oder dialektale Sprache attestiert, werden sie nicht ermutigt, die Sprache zu verwenden, sondern ihr eher entfremdet.

Das Werk Linguistic genocide or superdiversity untersucht den Wandel der Minderheitensprachen und ihrer Sprachgemeinschaften. Wie die Herausgeber Saarikivi und Toivanen in ihrem Vorwort feststellen, streben sie keine Dekonstruktion der traditionellen Sprachgemeinschaften an, sondern wollen zeigen, dass der Begriff Superdiversität jene neuen sprachlichen und ethnischen Identitäten widerspiegelt, die entstehen, wenn die alte Lebensweise der Sprachgemeinschaften schwindet. Insgesamt bietet das Werk eine neuartige Perspektive auf die Situation der uralischen Sprachen und deren Erforschung und öffnet ein neues Fenster für die Untersuchung des Wandels dieser Sprachen.

Sirkka Saarinen

\title{
Etymologische Erörterung ungarischer Wörter
}

\section{$<$ https://doi.org/| 0.33339/fuf.7954|>}

László Hontr: A magyar és a nyugati ótörök szókészleti kapcsolatairól [Über die lexikalischen Beziehungen zwischen dem Ungarischen und dem Westalttürkischen]. Segédkönyvek a nyelvészet tanulmányozásához 196. Budapest: Tinta könyvkiadó 2017. 218 S.
László Honti, der Grand Old Man der Ostjakologie und der finnischugrischen Sprachgeschichte, ließ sich durch das 2011 von András Róna-Tas und Árpád Berta veröffentlichte zweibändige Wörterbuch (West Old Turkic. Turkic loanwords in Hungarian) dazu anregen, eine Stellungnahme zu den in diesem 
Wörterbuch als ungesichert bezeichneten türkischen Etymologien zu verfassen. Hontis Erörterung beschränkt sich nicht auf die türkischen Hypothesen der erwähnten Wörter, sondern er behandelt diese fast 50 Wörter gründlich und betrachtet ihre gesamte Forschungsgeschichte mit allen Quellen. Jedes Wort erhält ein eigenes Kapitel, in dessen erstem Teil er detailliert alles durchgeht, was in der etymologischen Literatur jemals darüber geschrieben wurde, während der zweite Teil aus seinen eigenen Kommentaren und deren Resultat besteht. Ein Teil der Wörter kann seiner Ansicht nach als türkische Entlehnung gelten, für einen Teil findet sich eine andere Etymologie oder ihre Herkunft bleibt unbekannt.

In Ungarn wurden in der zweiten Hälfte des 20. Jahrhunderts vier bedeutende etymologische Wörterbücher veröffentlicht: A magyar nyelv történeti-etimológiai szótára (= TESz 1-4, 1967-1984), A magyar szókészlet finnugor elemei (= MSzFE 1-4, 1967-1981), Uralisches etymologisches Wörterbuch (= UEW 1-3, 1988, in dessen Redaktion Honti selbst mitwirkte) und Etymologisches Wörterbuch des Ungarischen (= EWUng 1-3, 1993-1997). MSzFE und UEW wurden vorwiegend von Finnougristen verfasst, TESz und EWUng eher von Hungarologen. In gleichaltrigen Wörterbüchern
(TESz vs. MSzFE, UEW vs. EWUng) gelangt man (auch bei den in Hontis Werk erwähnten Wörtern) erstaunlich oft zu völlig unterschiedlichen Herkunftserklärungen, für ein Wort können also, je nach dem jeweiligen Wörterbuch, mehrere verschiedene Ursprünge postuliert werden.

Unter den Wörtern, die in Hontis Werk behandelt werden, sind viele hochfrequente Wörter, deren Herkunft unklar ist. Beispielsweise wurde das Wort úr 'Herr' (und das nach dem Vorbild des Deutschen und der slavischen Sprachen davon abgeleitete ország 'Staat') als iranisch, türkisch und finnisch-ugrisch betrachtet. Honti zufolge kann úr als Verschmelzung eines auf die finnischugrische Ursprache zurückgehenden Wortes und eines türkischen Lehnwortes gelten (S. 71-72). Zur selben Schlussfolgerung gelangt er auch in einigen anderen Fällen. Da die ungarischen Wörter lautlich abgeschliffen sind, sich also häufig auf nur eine Silbe verkürzt haben, sind unter ihnen viele Homonyme, d.h. die Grenzziehung zwischen Homonymie und Polysemie ist von zentraler Bedeutung. Die lautliche Abgeschliffenheit erschwert auch die Konstruktion unbestreitbarer etymologischer Erklärungen.

Hontis Werk enthält zahlreiche interessante Beobachtungen. Er erklärt u.a., weshalb sajtó 'Presse; 
Zeitung' etymologisch derselben Wortfamilie zugeordnet werden könnte wie sajt 'Käse' (50-51), weist nach, dass ungarisch táltos 'Seher, Magier' ugrischer (und nicht türkischer) Herkunft ist (62-67), und legt dar, wie die Stammformen der Wörter mese 'Märchen; Rätsel' und magy(ar) 'ungarisch' durch Kontamination und semantische Entwicklung zusammengefallen sind (dieses Kapitel ist das längste des Werks: 129-147).

Ein separates Kapitel widmet Honti der Vertretung der Sibilanten der Ursprache in den heutigen ugrischen Sprachen (168-178). In einem eigenen Kapitel erörtert er auch das mögliche Vorkommen der Geminat-Affrikate bereits in der finnisch-ugrischen Ursprache (178-188). Unetymologische Konsonanten (hauptsächlich das in einigen Wörtern der ungarischen Gegenwartssprache vorkommende g) behandelt Honti im Kapitel 32 und dessen Unterkapiteln (101-117). Er führt das rätselhafte $g$ in den Akkusativformen des 1. und 2. Personalpronomens im Ungarischen (engem, téged) auf ${ }^{\star} \eta k$ zurück, auf dasselbe betonende Suffix, das den Personalpronomina im Mansischen angefügt wird.

Hontis Werk kann über die vorgelegten Ergebnisse zur Wortgeschichte hinaus als gutes Lehrmaterial der etymologischen Forschung gelten: Zusätzlich zur Analyse der Lautgestalt muss man die verwandten Sprachen und ihre Dialekte einbeziehen. Man muss auch die Ableitungen und Komposita betrachten, sofern sie zusätzliche Informationen über die Bedeutung des Wortes bieten (vgl. z.B. den Vergleich identischer Ableitungstypen im Zusammenhang mit den Verben gyúl 'sich entzünden' und gyújt 'anzünden' [37-38]). Man muss andere (verwandte) Sprachen und die dort begegnenden Parallelen vor allem bei der Entwicklung der Semantik der Wörter untersuchen. Auch in frühen Untersuchungen kann man gelegentlich Hinweise und wertvolle Informationen finden, darf also auch sie nicht geringschätzen. Etymologische Forschung erfordert die Genauigkeit und Geduld eines Uhrmachers. In vielen Fällen ist das Ergebnis dieser Arbeit ein Puzzlespiel, in dem jedes Teil seinen Platz findet, d.h. die Herkunft des Wortes wird geklärt. Nicht immer gelangt man anhand des verfügbaren Materials zu einer eindeutigen Lösung. Hontis Werk bietet begründete Lösungen für viele etymologische Rätsel. Gleichzeitig führt es den Leser auf interessante Forschungsreisen in die Geschichte der Wörter und ihrer Bedeutungen.

Sirkka Saarinen 


\section{Literatur}

EWUng = Etymologisches Wörterbuch des Ungarischen 1-3. Hrsg. Loránd Benkő. Akadémiai Kiadó. Budapest 1993-1997.

MSzFE = A magyar szókészlet finnugor elemei I-IV. Hrsg. György Lakó. Akadémiai Kiadó. Budapest 1967-1981.

RónA-TAs, ANDrÁs \& ÁrpÁd Berta: West Old Turkic. Turkic loanwords in Hungarian. Part 1: Introduction, Lexicon: "A-K". Turcologica 84. Harrassowitz Verlag. Wiesbaden 2011.
RónA-TAs, ANDrÁs \& ÁrpÁD BERTA: West Old Turkic. Turkic loanwords in Hungarian. Part 2: " $L-Z$ ". Conclusions, Apparatus. Turcologica 84. Harrassowitz Verlag. Wiesbaden 2011. $\mathrm{TESz}=$ A magyar nyelv történeti-etimológiai szótára 1-4. Hrsg. Loránd Benkő. Akadémiai Kiadó. Budapest 19671984.

UEW = Uralisches etymologisches Wörterbuch I-III. Hrsg. Károly Rédei. Akadémiai Kiadó - Otto Harrassowitz. Budapest - Wiesbaden 1988.

\section{Detachment constructions in European languages and beyond}

M. M. Jocelyne Fernandez-Vest: Detachments for Cohesion. Toward an Information Grammar of Oral Languages. Empirical Approaches to Language Typology 56. De Gruyter Mouton 2015. XVII + 290 pp.

The book, as its title illustrates, examines Detachment Constructions in oral languages from various perspectives. The term oral languages includes both strictly oral languages without a written tradition and the spoken variants of written languages. The use of Detachment Constructions is presented in several different discourse styles crosslinguistically, comparing European languages belonging to the IndoEuropean and the Finno-Ugric groups, and also considering other languages briefly.

Dislocated NPs either in front or at the end of utterances are traditionally referred to as detachments (or dislocations). Detachment Constructions are used mainly in spoken languages as a device for information structuring. They usually mark the topic when it is inactive in the hearer's consciousness. (Lambrecht 1994, 181-184.)

The author distinguishes Initial and Final Detachments and introduces previous research to exemplify their different functions. Initial Detachments usually share 